\title{
THERMAL MECHANICALLY COUPLED FINITE ELEMENT ANALYSIS IN METAL-FORMING PROCESSES
}

\author{
J. van der LUGT and J. HUETINK \\ Twente University of Technology, Department of Mechanical Engineering, Division Production. \\ P.O. Box 217, 7500 AE Enschede, The Netherlands \\ Received 15 November 1984 \\ Revised manuscript received 10 June 1985
}

\begin{abstract}
A combined Eulerian-Lagrangian finite element formulation is presented for the analysis of metal-forming, coupled with thermal effects. The procedure developed involves incrementally solving a coupled set of equations for both the displacement and the temperature. The material properties may be temperature-dependent. The procedure has been applied to the upsetting and the wire-drawing process.
\end{abstract}

\section{Introduction}

In metal-forming processes such as rolling, extrusion and stamping, large plastic deformation as well as large temperature changes may occur. The temperature changes of the body are either due to external heating, or to the conversion of mechanical work into heat through plastic deformation or friction.

Since the material properties depend on the temperature, temperature changes have a significant influence on the deformation process. At the same time a nonhomogeneous temperature distribution can, after cooling. lead to undesirable residual stress or deformation. A thermal mechanically coupled analysis is necessary to model the physical phenomena observed. In this paper a finite element technique is presented using a combined EulerianLagrangian approach to incrementally solve the large displacement elastic-plastic problem, coupled with the thermal problem. In relation to previously developed and reported work on the treatment of thermomechanically coupled deformation processes, [1-3], the combined Eulerian-Lagrangian approach has the advantage of easily defining boundary conditions depending on the spatial location but not on the material flow. An even more important advantage of this approach is that in metal-forming processes workpiece and tool can be analyzed simultaneously because nodal points of both parts may be chosen in such a way that they keep-independently from slip occurring-equal spatial coordinates. Hence degrees of freedom can easily be connected.

\subsection{Notation}

Throughout the paper, index notation with summation convention and Kronecker delta $\left(\delta_{i j}\right)$ are used. The theory is formulated in terms of rectangular Cartesian coordinates and Cartesian 
tensors. The superposed dot is employed for the material time derivative in the moving continuum:

$$
\dot{A}=\frac{\partial A}{\partial t}+\frac{\partial A}{\partial x_{i}} \dot{x}_{i}
$$

where $\dot{x}_{i}=v_{i}$ (the velocity). The partial derivative of a function $f_{t}$ with respect to the coordinates $x_{j}$ is given by

$$
\partial f_{i} / \partial x_{j}=f_{i, j} .
$$

\section{The constitutive law in coupled thermomechanical problems}

The theory is restricted to time-independent, elastic-plastic, isotropic material properties. The stress-strain relation for small reversible deformations can be deduced from the expression of free energy, [4-8]. The linear form of this relation is expressed by [9]:

$$
\sigma_{i j}=E_{i j k l} \varepsilon_{k l}+L_{i j} \theta
$$

where $\sigma_{i j}$ is the Cauchy stress tensor, $\varepsilon_{i j}$ the Euler-Almansi strain tensor, $E_{y k l}$ the elastic tensor, $L_{i j}$ the thermal tensor and $\theta$ the relative temperature:

$$
\begin{aligned}
& E_{i j k l}=\frac{E}{2(1+\nu)}\left(\delta_{i l} \delta_{j k}+\delta_{i k} \delta_{j l}+\frac{2 \nu}{1-2 \nu} \delta_{i j} \delta_{k l}\right), \\
& L_{i j}=-\frac{E \alpha}{1-2 \nu} \delta_{i j}
\end{aligned}
$$

with $\alpha$ as the coefficient of linear expansion,

$$
\theta=T-T_{0}
$$

with $T$ as the absolute temperature and $T_{0}$ as the initial temperature.

If plastic flow occurs the total strain can be decomposed into a reversible part, the elastic and thermal strain, and an irreversible part, the plastic strain:

$$
\varepsilon_{i j}=\varepsilon_{i j}^{\mathrm{rv}}+\varepsilon_{i j}^{\mathrm{pl}} .
$$

The plastic strain is not related to the stress, there is only a relation between the stress rate and the rate of deformation $\left(d_{i j}\right)$. The rate of deformation is defined as:

$$
d_{i j}=\frac{1}{2}\left(v_{i, j}+v_{j, i}\right) \text {. }
$$

The rate of deformation can also be decomposed into a reversible part and a plastic part: 


$$
d_{i j}=d_{i j}^{\mathrm{rv}}+d_{i j}^{\mathrm{pl}} .
$$

The relation between the stress rate and the rate of deformation can be expressed by means of the Jaumann derivative of the stress. The Jaumann derivative of the stress for small elastic deformations under isothermal conditions is expressed by [10]

$$
\stackrel{\nabla}{\sigma}_{i j}=E_{i j k l} d_{k l}^{\mathrm{rv}}
$$

In coupled thermomechanical problems equation (5) can be extended, using (1), to

$$
\stackrel{\nabla}{\sigma}_{i j}=\stackrel{\nabla}{E}_{i k l} \varepsilon_{k l}^{\mathrm{rv}}+E_{i j k l} d_{k l}^{\mathrm{rv}}+\stackrel{\nabla}{L}_{i j} \theta+L_{\imath j} \dot{\theta}
$$

The yield surface bounds the region in the stress space in which for any stress vector only elastic strain occurs. The mathematical description of the yield surface is given by

$$
\varphi\left(\sigma_{i j}, H, \theta\right)=0,
$$

where $\varphi$ is the yield function, a function of the stress $\left(\sigma_{i j}\right)$, the history of plastic deformation $(H)$ and the temperature $(\theta)$. For plastic flow to occur $\varphi$ must be identical to zero,

$$
\dot{\varphi}=\frac{\partial \varphi}{\partial \sigma_{i j}} \dot{\sigma}_{i j}+\frac{\partial \varphi}{\partial H} \dot{H}+\frac{\partial \varphi}{\partial \theta} \dot{\theta}=0 .
$$

Isotropic hardening yields:

$$
\dot{H}=\dot{\tilde{\varepsilon}}^{\mathrm{pl}}=\left(\frac{2}{3} d_{i j}^{\mathrm{pl}} d_{i j}^{\mathrm{pl}}\right)^{1 / 2},
$$

where $\tilde{\varepsilon}^{\mathrm{pl}}$ is the equivalent plastic strain.

The plastic deformation rate is found by using the Drucker postulate [11]

$$
d_{i j}^{\mathrm{pl}}=\mu \partial \varphi / \partial \sigma_{i j} .
$$

Substitution of (9) and (10) in (8) results in:

$$
\frac{\partial \varphi}{\partial \sigma_{i j}} \dot{\sigma}_{i j}+\mu \frac{\partial \varphi}{\partial H}\left(\frac{2 \partial \varphi}{3 \partial \sigma_{i j}} \frac{\partial \varphi}{\partial \sigma_{i j}}\right)^{1 / 2}+\frac{\partial \varphi}{\partial \theta} \dot{\theta}=0 .
$$

Isotropic materials yield

$$
\frac{\partial \varphi}{\partial \sigma_{k l}} \dot{\sigma}_{k l}=\frac{\partial \varphi}{\partial \sigma_{k l}} \stackrel{\nabla}{\sigma}_{k l}
$$

By eliminating $\mu$ and the plastic deformation rate, the Jaumann derivative (6) can be written as 
where

$$
\stackrel{\nabla}{\sigma}_{\imath \jmath}=\left\{E_{i j k l}-(1-h) Y_{i j k l}\right\} d_{k l}+\left\{D_{i j k l}-(1-h) Y_{i j k l}^{*}\right\} \varepsilon_{k l}^{\mathrm{rv}} \dot{\theta}+\left\{K_{i j}-(1-h) Y_{l j}^{\theta}\right\} \dot{\theta},
$$

$$
\begin{aligned}
& D_{i j k l}=\frac{\mathrm{d} E}{\mathrm{~d} \theta} \frac{1}{2(1+\nu)}\left(\delta_{i l} \delta_{j k}+\delta_{i k} \delta_{j t}+\frac{2 \nu}{1-2 \nu} \delta_{i j} \delta_{k l}\right), \\
& K_{i j}=-\left(\frac{\mathrm{d} E}{\mathrm{~d} \theta} \frac{\alpha}{(1-2 \nu)} \theta+\frac{E \alpha}{1-2 \nu}\right) \delta_{i j} .
\end{aligned}
$$

The Von Mises yield condition has been applied:

$$
\varphi=\frac{1}{2} s_{i j} s_{i j}-\frac{1}{3}\left(\sigma_{y}\left(\tilde{\varepsilon}^{\mathrm{pl}}, \theta\right)\right)^{2},
$$

where $s_{i j}$ is the stress deviator and $\sigma_{y}$ is the yield stress depending on the equivalent plastic strain $\left(\tilde{\varepsilon}^{\mathrm{pl}}\right)$ and the temperature $(\theta)$. The yield tensors can be written as:

$$
Y_{i j k l}=\frac{s_{i j} E s_{k l}}{\frac{2}{3}\left(\sigma_{y}\right)^{2}(1+\nu)}, \quad Y_{i j k l}^{*}=\frac{s_{i j}(\mathrm{~d} E / \mathrm{d} \theta) s_{k l}}{\frac{2}{3}\left(\sigma_{y}\right)^{2}(1+\nu)}, \quad Y_{i j}^{\theta}=-\frac{\partial \sigma_{y}}{\partial \theta} \frac{s_{i j}}{\sigma_{y}} .
$$

Poisson's ratio $(\nu)$ is assumed to be temperature independent. The hardening coefficient $h$ will be

$$
h=\frac{\frac{1}{3} \partial \sigma_{y} / \partial \tilde{\varepsilon}^{\mathrm{pl}}}{G+\frac{1}{3} \partial \sigma_{y} / \partial \tilde{\varepsilon}^{\mathrm{pl}}}
$$

\section{The finite element technique}

The finite element technique applied to coupled thermomechanical problems is based on mechanical as well as thermal equilibrium.

\subsection{The mechanical equilibrium}

The basic equations are given by the equilibrium of forces:

$$
\sigma_{i j, j}+F_{i}=0 .
$$

The so-called 'weak form' of the equilibrium of forces, when applying the divergence theorem, yields

$$
\delta W=\int_{V} \sigma_{i j} \delta d_{i j} \mathrm{~d} V-\int_{V} F_{i} \delta v_{i} \mathrm{~d} V-\int_{S} T_{i} \delta v_{i} \mathrm{~d} S=0, \quad \forall \delta v_{i},
$$

where $\delta v_{i}$ is the virtual velocity. In order to obtain an expression in which the relation between 
the stress rate and the deformation rate (13) can be substituted, we must take the material time derivative of the weak form. The material time derivative of the weak form under isothermal conditions is given by J. Huetink [12]. Similarly the material time derivative of the weak form under non-isothermal conditions can be derived. This results in:

$$
\begin{aligned}
\delta \dot{W}= & \int_{V}\left\{\left(E_{i j m n}^{*} d_{m n}+D_{i j k l}^{*} \sigma_{k l} \dot{\theta}+K_{i j}^{*} \dot{\theta}-2 \sigma_{k j} d_{i k}\right) \delta d_{i j}\right. \\
& \left.+\sigma_{i j} v_{k, j} \delta v_{k, i}\right\} \mathrm{d} V-\int_{V} \dot{F}_{i} \delta v_{i} \mathrm{~d} V-\int_{S} \dot{T}_{i} \delta v_{\imath} \mathrm{d} S=0, \quad \forall \delta v_{\imath},
\end{aligned}
$$

where

$$
\begin{aligned}
& E_{i j m n}^{*}=E_{i j m n}-(1-h) Y_{i j m n}, \quad D_{i j k l}^{*}=\left(D_{i j m n}-(1-h) Y_{i j m n}^{*}\right) E_{k l m n}^{-1}, \\
& K_{i j}^{*}=K_{i j}-D_{i j m n} E_{k l m n}^{-1} L_{k l} \theta-(1-h)\left(Y_{i j}^{\theta}-Y_{i j m n}^{*} E_{k l m n}^{-1} L_{k l} \theta\right) .
\end{aligned}
$$

\subsection{The thermal equilibrium}

Based on the conservation of energy the thermal equilibrium can be expressed as

$$
\rho c \dot{\theta}-\lambda \theta_{, i i}-q=0,
$$

where $\rho$ is the mass density, $c$ is the specific heat (a function of the temperature), $\lambda$ is the coefficient of heat conduction and $q$ is the heat production per unit of volume. The weak form of the conservation of energy, when applying the divergence theorem, yields

$$
\delta \dot{Q}=\int_{V} \rho c \dot{\theta} \delta \dot{\theta} \mathrm{d} V+\lambda \int_{V} \theta_{, \imath}(\delta \dot{\theta})_{, i} \mathrm{~d} V-\int_{V} q \dot{\delta} \dot{\theta} \mathrm{d} V+\int_{S} \psi_{n} \delta \dot{\theta} \mathrm{d} S=0, \quad \forall \delta \dot{\theta},
$$

where $\psi_{n}$ is the heat flow, directed outwards in space and normal to the surface and $\delta \dot{\theta}$ is the virtual temperature rate. In thermomechanically coupled problems the heat production per unit of volume $(q)$ is assumed to be equal to the energy dissipated by the plastic deformation

$$
q=\sigma_{i j} d_{i j}^{\mathrm{pl}} .
$$

Using (8) and performing appropriate manipulations we can achieve an expression for the heat production per unit of volume:

$$
q=(1-h) \sigma_{i j}\left\{\frac{Y_{i j k l}^{*}}{2 G} E_{k l m n}^{-1} \sigma_{m n} \dot{\theta}+\frac{Y_{i j k l}}{2 G} d_{k l}+\frac{Y_{i j}^{\theta}}{2 G} \dot{\theta}\right\}
$$

Substituting this relation into the weak form (19) we obtain:

$$
\begin{aligned}
\delta \dot{Q}= & \int_{V} \rho c \dot{\theta} \dot{\theta} \dot{\theta} \mathrm{d} V+\lambda \int_{V} \theta_{, \imath}(\delta \dot{\theta})_{, \imath} \mathrm{d} V \\
& -\int_{V} \sigma_{i j}\left(Y_{\imath j k l}^{* * *} \sigma_{k l} \dot{\theta}+Y_{i j k l}^{* *} d_{k l}+Y_{i,}^{\theta \theta} \dot{\theta}\right) \delta \dot{\theta} \mathrm{d} V-\int_{S} \psi_{n} \delta \dot{\theta} \mathrm{d} S=0, \quad \forall \delta \dot{\theta},
\end{aligned}
$$


where

$$
\begin{aligned}
& Y_{i j k l}^{* *}=(1-h) \frac{Y_{i \jmath k l}}{2 G}, \quad Y_{\imath \jmath k l}^{* * *}=(1-h) \frac{Y_{i j m n}^{*}}{2 G} E_{k l m n}^{-1}, \\
& Y_{\imath !}^{\theta \theta}=(1-h)\left(\frac{Y_{i j}^{\theta}}{2 G}-\frac{Y_{l j m n}^{*}}{2 G} E_{k l m n}^{-1} L_{k l} \theta\right) .
\end{aligned}
$$

\subsection{The weak form of coupled thermomechanical problems}

In coupled thermomechanical problems mechanical equilibrium as well as thermal equilibrium have to be satisfied. The weak form of coupled problems $(\delta \dot{P})$ becomes

$$
\delta \dot{P}=\delta \dot{Q}+\delta \dot{W}=0, \quad \forall \delta v_{\imath}, \delta \dot{\theta}
$$

\subsection{The set of equations for the finite element solution}

Isoparametric elements with Gauss numerical integration [13] have been applied. The nodal degrees of freedom are the components of the displacement rate and the temperature rate. The same interpolation functions are used to express the temperature rate and the displacement rate. However, a modification has been applied to the interpolation and numerical integration of the temperature in order to avoid inaccurate temperature prediction in the case of fast deformation. In that case, the contribution of conduction to the temperature rate is very small compared to the contribution of dissipation. These undesired numerical effects that give inaccurate temperature predictions are illustrated by means of a one-dimensional problem, without heat transfer. The temperature distribution is shown in Fig. 1(a). One of the boundary nodal points is subjected to a prescribed jump in temperature. It shows that the other nodal point temperatures also change.

The coupling between the nodal point temperature rates will cause a virtual heat flow, not as a result of heat transfer but because of the position of the integration points and the value of the interpolation function at these points. By shifting the position of the integration points to the position of the corresponding nodal points the coupling between the nodal points, which is not due to heat transfer, will vanish. This can be regarded as an orthogonalization of the interpolation functions:

$$
\int_{x} \Psi^{K}(x) \Psi^{L}(x) \mathrm{d} x=\sum_{\mathrm{ip}=1}^{N} \Psi^{K}\left(x_{1 \mathrm{p}}\right) \Psi^{L}\left(x_{1 \mathrm{p}}\right)=\delta_{K L},
$$

where $\Psi^{K}, \Psi^{L}$ are the interpolation functions, related to nodal points $K$ and $L$ respectively. Subscript ip stands for integration point and $N$ is the number of integration points. By applying the modified integration on the above-mentioned system with the same boundary conditions, the temperature is distributed as shown in Fig. 1(b). Except for the temperature prescribed, the nodal temperatures remain unchanged. The results of the modified integration applied to the temperature problem are more accurate. Therefore, it is justifiable to use the orthogonalization of the interpolation functions for the temperature rate. The inaccuracy of the temperature prediction using the Gauss numerical integration is illustrated by the numerical analysis of the upsetting problem of T.B. Wertheimer [3]. The undesired numerical 

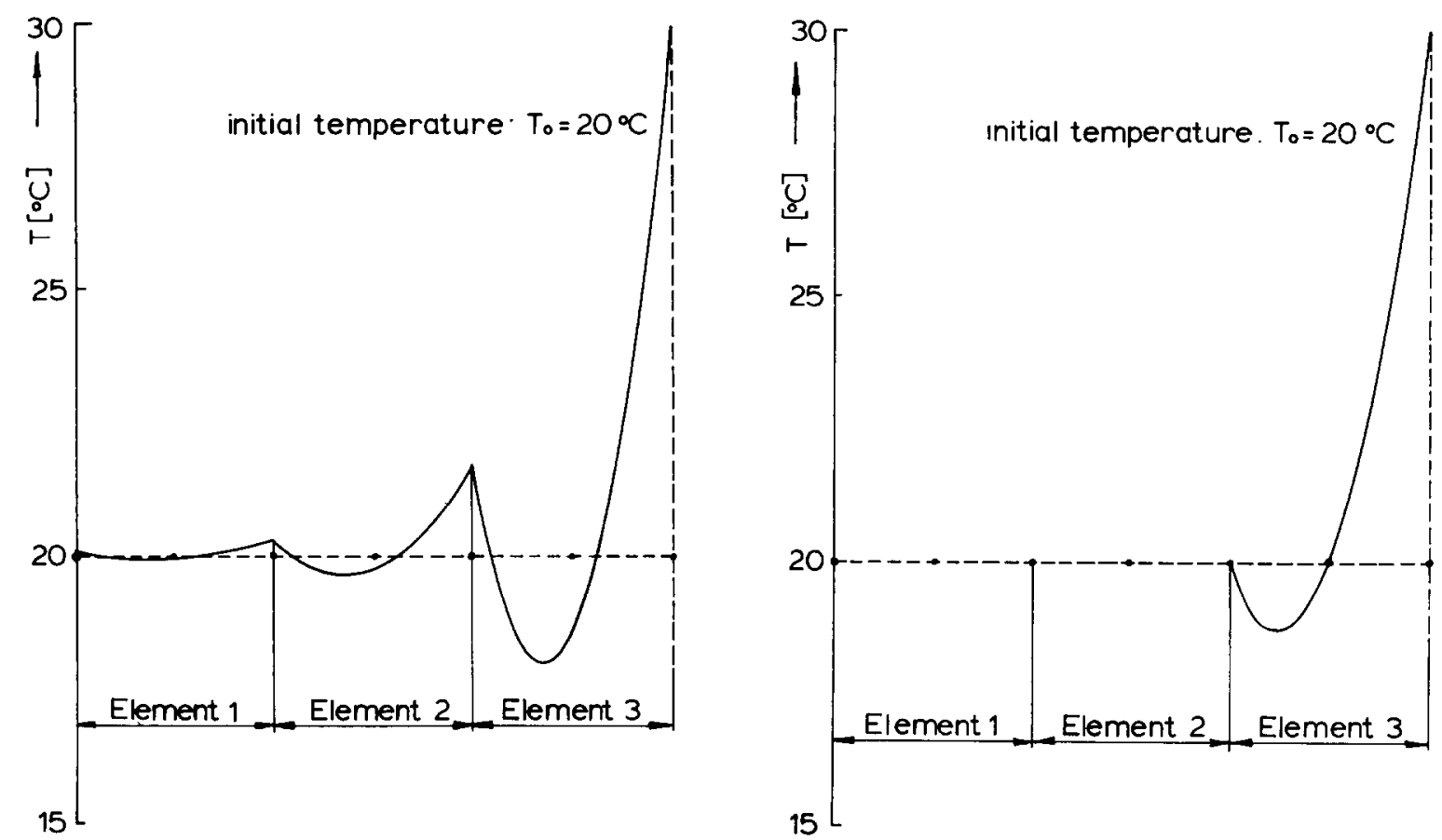

Fig. 1. Temperature distribution for a one-dimensional problem. (a) Using the Gauss numerical integration. (b) Using the modified numerical integration.

effects show up most clearly by low temperatures in the area where no plastic deformation occurs. Before the integration was modified, we carried out an analysis of the same upsetting problem and found results similar to those calculated by Wertheimer. The velocity interpolation functions remain unchanged.

Now, the weak form for coupled thermomechanical problems results in:

$$
[K] v=R,
$$

where $[K]$ is the nonsymmetric stiffness matrix, $v$ is the vector containing all nodal point quantities and $\boldsymbol{R}$ is the load vector.

\subsection{Solving the set of equations}

By using the Crout factorization the stiffness matrix can be written as the product of an upper triangular matrix with unit diagonal and a lower triangular matrix. The triangular decomposition of the stiffness matrix allows the set of equations to be solved in two steps, the 'forward elimination' and the 'back-substitution' [14]. The nonsymmetric stiffness matrix has a symmetric structure. Therefore it is possible to reduce the required storage and computational effort by storing only the elements of the stiffness matrix within the local bandwidth. This was noted for symmetric matrices by Zienkiewicz [13] and Bathe and Wilson [15].

If, at time $t$, the shape, state of stress and boundary conditions are known, the nodal point velocities and temperature rates can be solved from (25). An implicit scheme is applied to the 
thermal part of the load vector. Within a time increment $\Delta t$ the material displacement increments, related to the nodal points, are approximated by:

$$
\Delta u_{i}^{N}=v_{i}^{N} \Delta t
$$

Similarly the temperature increments are approximated by

$$
\Delta \theta^{N}=\dot{\theta}^{N} \Delta t
$$

This method is identical to the updated Lagrange method $[16,17]$; the nodal points are coupled with the material points. Using a combined Eulerian-Lagrangian method the nodal point displacements do not coincide with the material displacement. This combined method requires additional calculations to the updated Lagrange method. Say that the displacement of nodal point $N$ is given by $\Delta x_{i}^{N}$ and the initial displacement and displacement increment of the material point of the step are represented by $u_{0 i}^{N}$ and $\Delta u_{i}^{N}$ respectively. The difference between the new location of the nodal point $N$ and the new location of the material point is (see Fig. 2)

$$
\Delta y_{i}^{N}=\Delta x_{i}^{N}-\Delta u_{i}^{N} .
$$

The new location of a material point that coincides with an internal point $x_{0,}$ of element $(e)$ at time $t$ is found by

$$
x_{1 \iota}=x_{0 i}+\Psi^{(e) N}\left(x_{0 i}\right) \Delta u_{i}^{N},
$$

where $\Psi^{(e) N}$ is the interpolation function, related to nodal point $N$ of element $(e)$. The new location of the corresponding element point is

$$
x_{2 i}=x_{0 i}+\Psi^{(e) N}\left(x_{0 i}\right) \Delta x_{i}^{N} .
$$

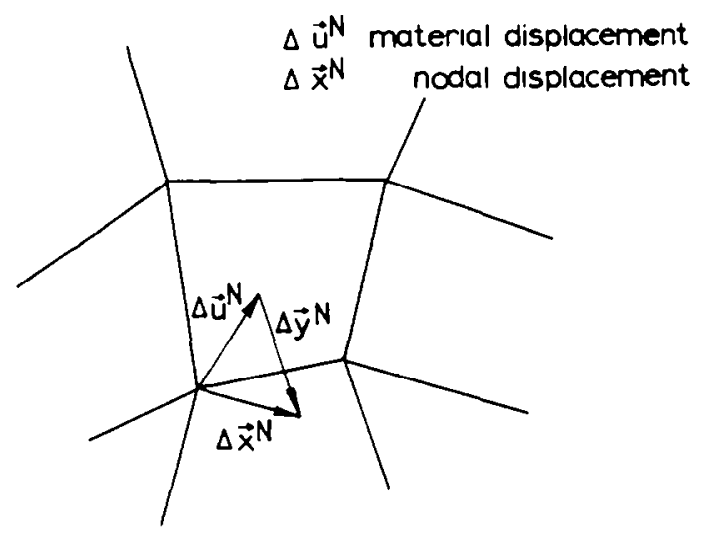

Fig. 2. Uncoupled nodal and material displacements. 
The distance between the points is

$$
\Delta y_{i}=x_{2 i}-x_{1 i}=\Psi^{(e) N}\left(x_{0 i}\right) \Delta y_{i}^{N} .
$$

The formulation of a combined Eulerian-Lagrangian method under isothermal conditions is given by J. Huetink [12]. The expression of the nodal displacements yields

$$
u_{i}^{N}=\Delta u_{i}^{N}+u_{0 i}^{N}+\frac{1}{\mathrm{Ne}}\left\{\sum_{e(N)} \Psi^{(e) M}\left(x_{0 i}^{N}\right)_{, k}\right\} u_{0 i}^{M} \Delta y_{k}^{N}
$$

where $\sum_{e(N)}$ indicates that the sum is taken over all elements that are connected to node $N$, and $\mathrm{Ne}$ is the number of elements connected to node $N$. The last term in the right-hand side of (32) accounts for convection of the material that flows through the elements. This term can be regarded as the linear term of a Taylor series expansion. If $\Delta x_{k}^{N}$ equals $\Delta u_{k}^{N}$, the last term in (32) vanishes and the formulation is equal to an updated Lagrange approach. If $\Delta x_{k}^{N}$ equals zero the formulation is a purely Eulerian approach, there is no element mesh distortion. By applying the Eulerian-Lagrangian method to coupled thermomechanical problems, the nodal temperatures can be calculated in the same way as the nodal displacements (32).

\subsection{The computer program}

The method described in the preceding sections has been implemented in the specialpurpose finite element program, DIEKA, developed by J. Huetink at the Twente University of Technology. The program has been applied to the analysis of an upsetting process and to the analysis of a wire-drawing process.

\section{Results}

\subsection{The upsetting process}

The upsetting proces is defined as the axial compression of an axisymmetric body between two perfectly rough, insulated plates. The material used is low carbon steel with code $\mathrm{Ck} 15$. The initial temperature is $20^{\circ} \mathrm{C}$, the initial height is $36 \mathrm{~mm}$ and the initial radius is $9 \mathrm{~mm}$. It is a closed system, there is no heat transfer to the environment (natural boundary condition). The mechanical material properties are obtained by tensile tests at several temperatures. The material properties are shown in Fig. 3. Only a quarter of the problem is modelled because of symmetry. The finite element discretization consists of 12 eight-noded quadratic, isoparametric elements. There are 102 displacement and 51 temperature degrees of freedom. The total imposed reduction was $(\Delta h / h) \cdot 100 \%=44 \%$, the loading time was 1.6 seconds. The analysis has been performed in 107 increments according to the updated Lagrange method. Fig. 4(a) shows the initial finite element discretization. In order to avoid unacceptable distortion of the elements a re-zoning has been applied. This re-zoning was made after step 43 by calculating 4 steps according to the combined Eulerian-Lagrangian method with $\Delta x_{i}^{N}(28)-(32)$ such that a more appropriate element mesh was achieved (Fig. 4(b)). No deformation occurs during the re-zoning steps ( $\Delta t=0, \Delta h=0)$. The final deformation is given by Fig. 4(c). 


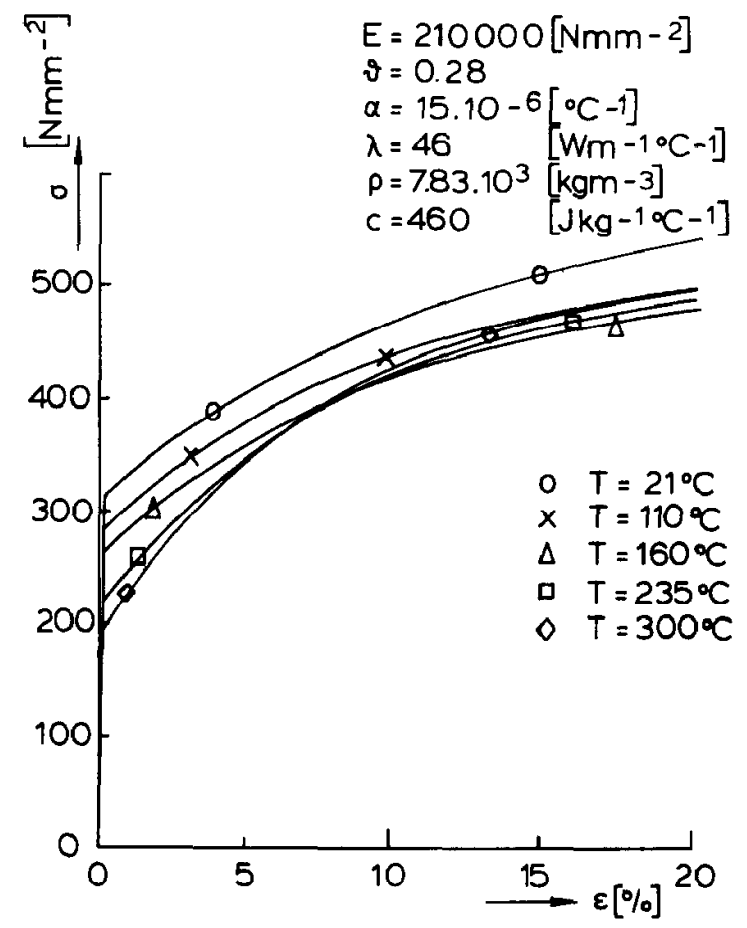

Fig. 3. The true-stress true-strain curve of the material used for the upsetting process, at several temperatures.

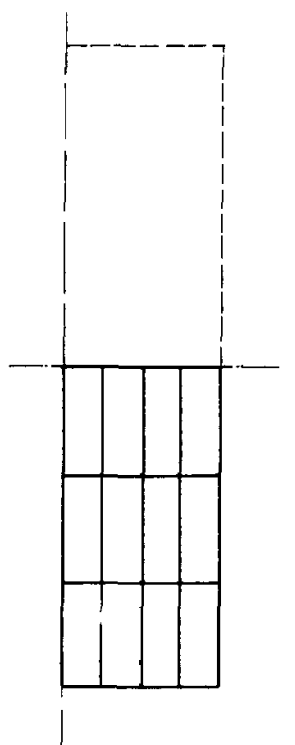

(a)

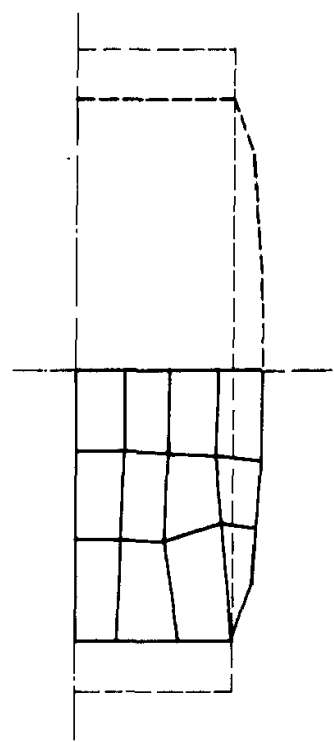

(b)

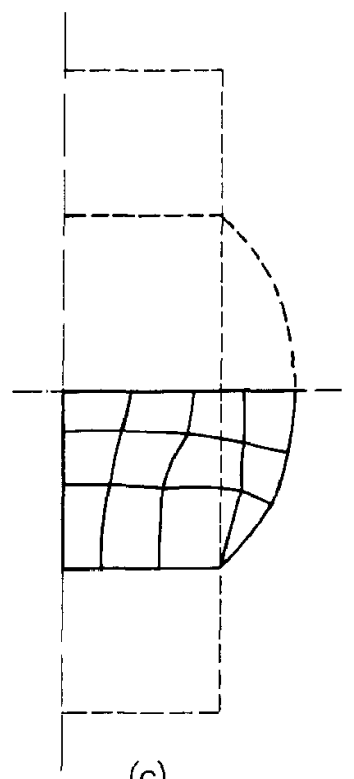

(c)

Fig. 4. (a) The initial finite element mesh. (b) The finite element mesh after re-zoning. (c) The finite element mesh at the last stage of the process. 
Fig. 5 shows the resulting temperature distribution for this model. Verification of the finite element model can be achieved by comparing the numerical results with the results obtained by experimental methods. The experiment is performed under the same conditions as mentioned above. The temperature has been measured, at medium height, on the outer surface of the billet. The results are given in Fig. 6 .

It can be seen that the boundary condition of perfect insulation is not achicved. Therefore it is necessary to correct the experimental results for heat transfer to the environment. For the heat transfer to the environment it is necessary to know the coefficient of heat transfer between billet and environment $(\beta)$. This coefficient follows from the expression of the heat flow $(\psi)$, towards the environment. When the billet has an almost uniform temperature $(\bar{\theta})$, the heat flow can be expressed by

$$
\psi=c p V \frac{\mathrm{d} \bar{\theta}}{\mathrm{d} t}=\beta\left(\bar{\theta}-\theta_{0}\right) \quad \text { or } \quad \beta=\frac{c \rho V \mathrm{~d} \bar{\theta} / \mathrm{d} t}{\bar{\theta}-\theta_{0}} .
$$

The temperature correction ( $\left.\theta_{\text {corr }}\right)$, at time $t$ and temperature $\theta$, can now be written as:

$$
\theta_{\text {corr }}=\frac{1}{c \rho V} \int_{0}^{t} \psi \mathrm{d} t \quad \text { or } \quad \theta_{\text {corr }}=\frac{\Delta \bar{\theta}}{\Delta t} \frac{1}{\left(\bar{\theta}-\theta_{0}\right)} \int_{0}^{t}\left(\theta-\theta_{0}\right) \mathrm{d} t .
$$

The experimental results, with the correction of the temperature for heat loss, are also given in Fig. 6. The loading time was fast. The heat transfer between billet and thermocouple is a slow process. This has to be taken into account when comparing the corrected experimental results with the numerical results. Therefore it is more reliable to compare the results in the steady

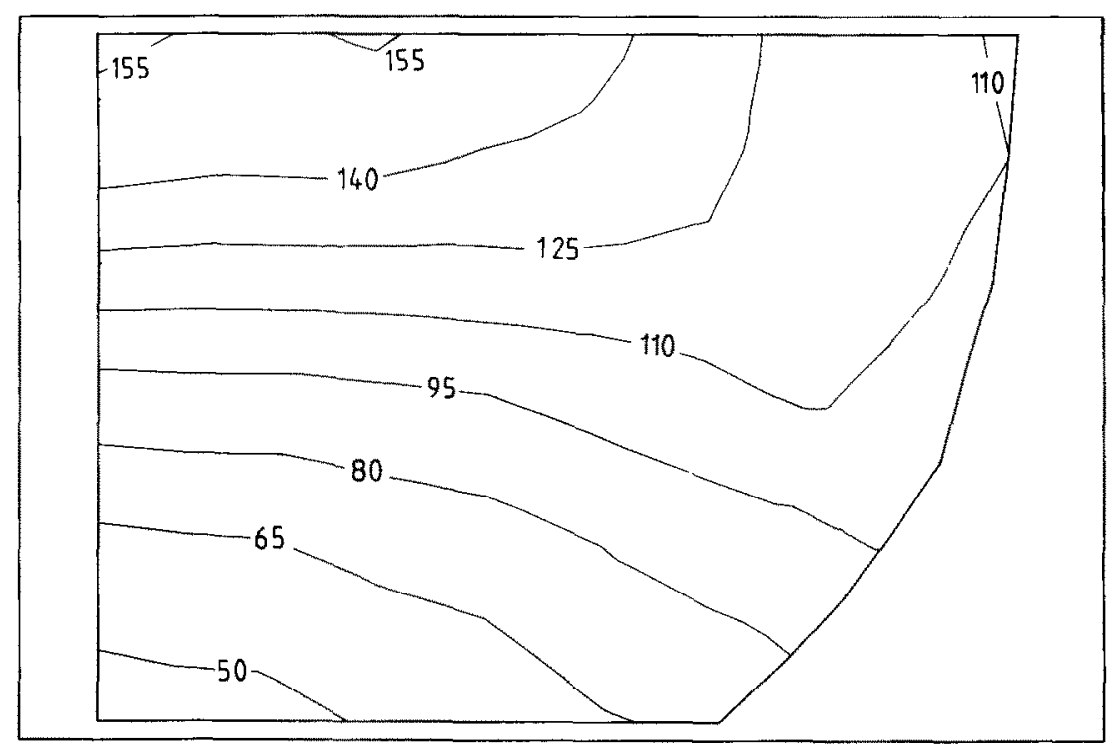

Fig. 5. The temperature distribution for the upsetting process. 


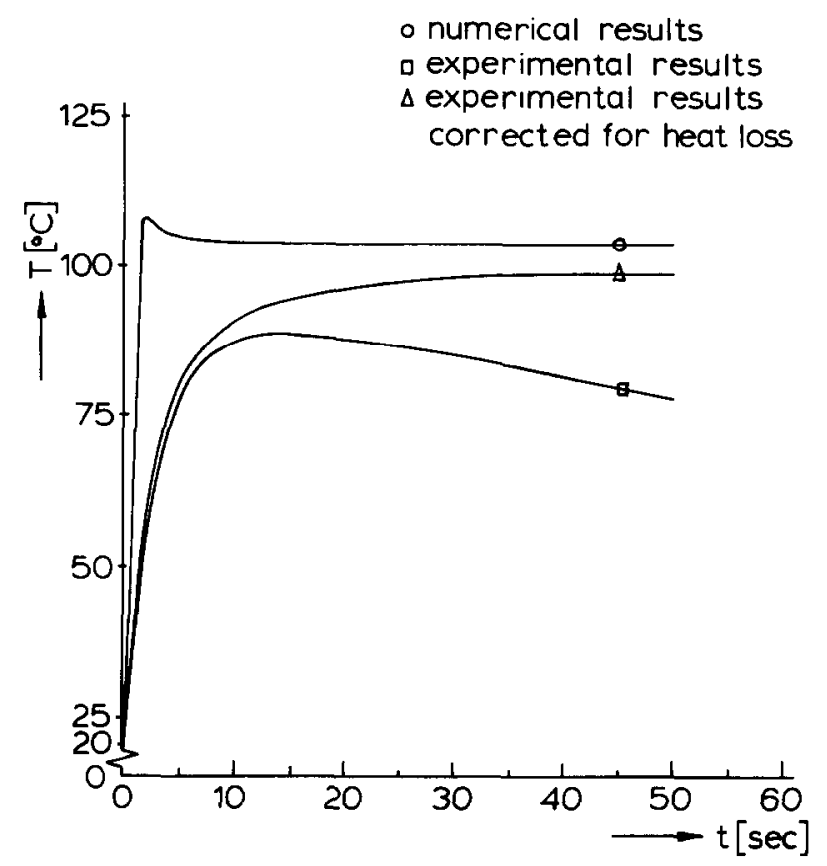

Fig. 6. Change in temperature, at medium height, on the outer surface of the billet, during and after the upsetting process.

state. For this purpose the numerical analysis has been continued with additional time steps and no deformation to achieve the steady state after the forming process. The results are given in Fig. 6. Now the numerical results and the (corrected) experimental results can be compared. The steady state temperature for the experiment was $100.5^{\circ} \mathrm{C}$, and for the numerical analysis $105.0^{\circ} \mathrm{C}$. The difference between these temperatures can be explained by the difference in respectively the measured and the calculated work that has been carried out (see Fig. 7). Slight differences may occur due to the modelling of the material used for the numerical analysis. The difference in work gives a difference in temperature of $4.5^{\circ} \mathrm{C}$; this coincides with the difference in temperature between the experiment and the numerical analysis.

\subsection{The wire-drawing process}

The model consists of a low carbon steel wire, drawn through a cobalt-bounded tungstencarbide die with a velocity of $1 \mathrm{~m} / \mathrm{s}$. The material properties are given in Fig. 8 and Table 1 respectively [18]. The initial temperature is $20^{\circ} \mathrm{C}$. There is no heat transfer to the environment (natural boundary condition). Only half of the problem is modelled because of symmetry. The problem geometry and element mesh are given in Fig. 9. A thin laycr between wire and dic, subjected only to shear, approximates the sticking friction. The value of the yield stress of this layer defines the extent to which sticking friction occurs. The yield stress of the layer in the conic section of the die in four-tenths of the yield stress of the wire at $20^{\circ} \mathrm{C}$ (initial temperature); the yield stress in the cylindric section is two-tenths of the yield stress of the wire at $20^{\circ} \mathrm{C}$. The material of the layer shows no hardening. The remaining material 


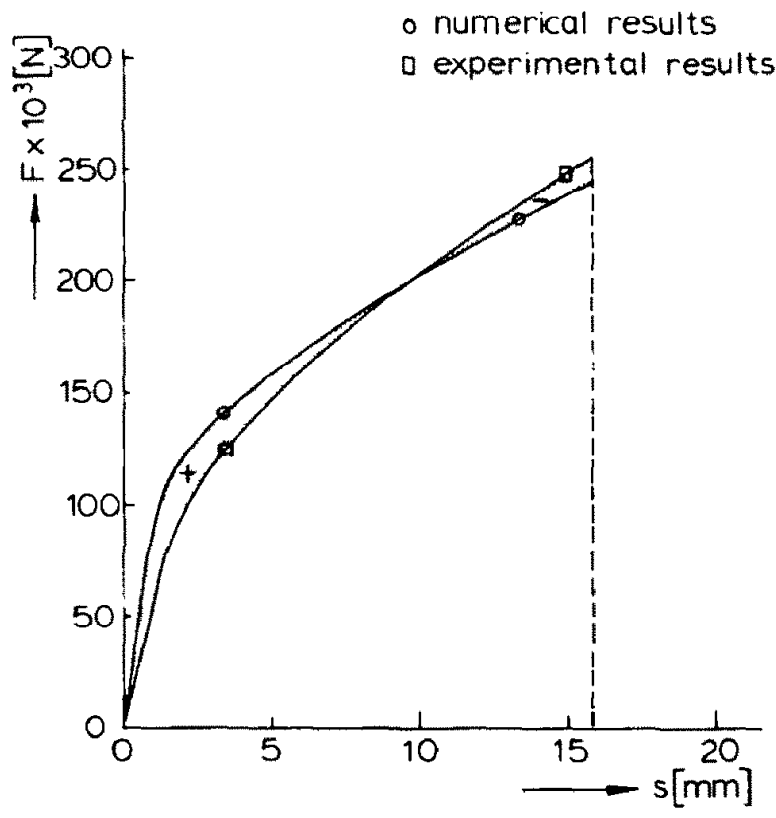

Fig. 7. Load displacement curve of the upsetting process.

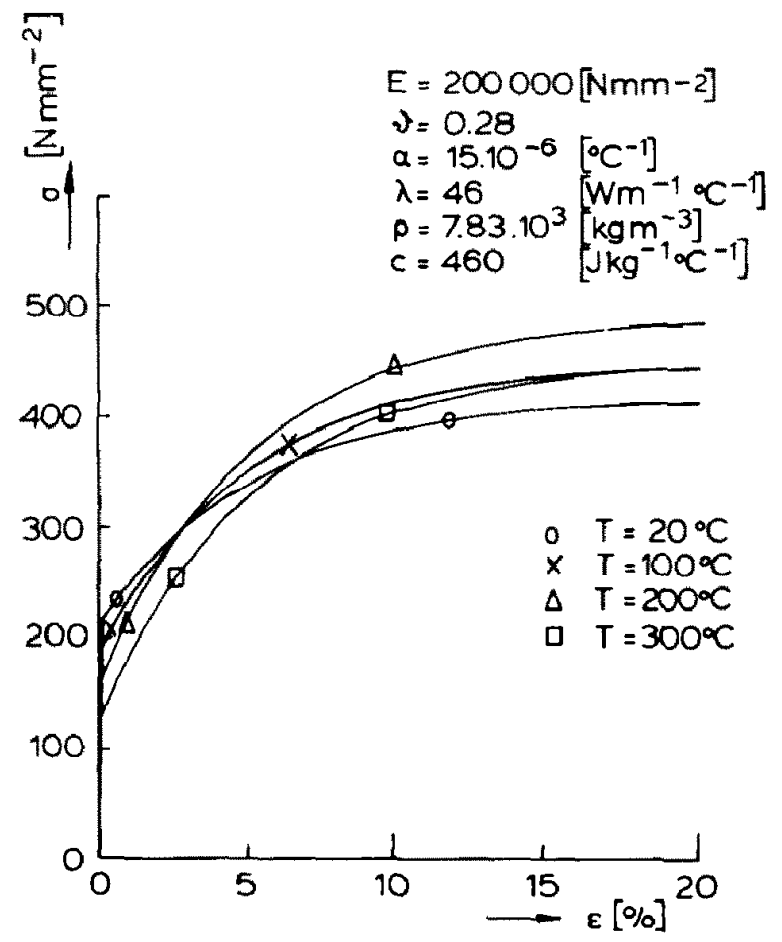

Fig. 8. The true-stress true-strain curve of the wire material, at several temperatures. 
Table 1

Material properties of the die

\begin{tabular}{cccccc}
\hline$E\left[\mathrm{Nmm}^{-2}\right]$ & $\nu$ & $\alpha\left[{ }^{\circ} \mathrm{C}^{-1}\right]$ & $\lambda\left[\mathrm{Wm}^{-1}{ }^{\circ} \mathrm{C}^{-1}\right]$ & $\rho\left[\mathrm{kgm}^{-3}\right]$ & $c\left[\mathrm{Jkg}^{-1}{ }^{\circ} \mathrm{C}^{-1}\right]$ \\
\hline 600000 & 0.28 & $5 \cdot 10^{-6}$ & 60 & $140 \cdot 10^{3}$ & 140 \\
\hline
\end{tabular}

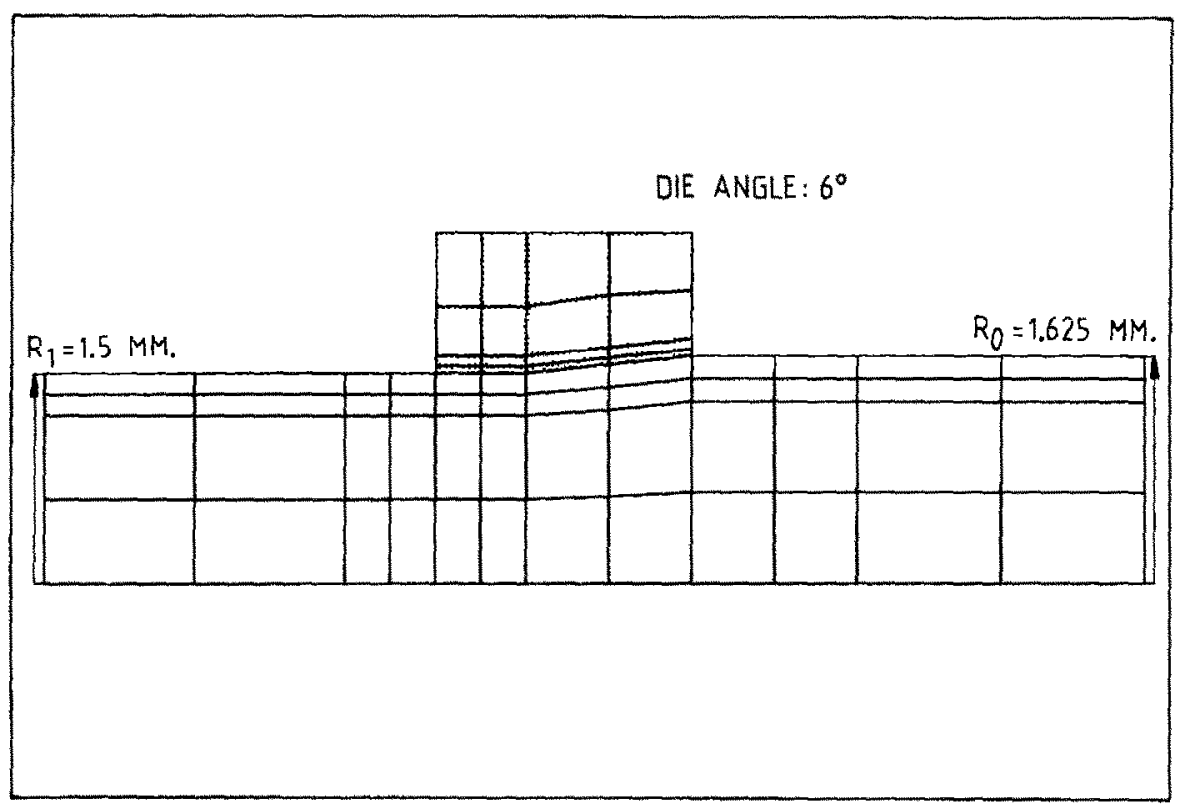

Fig. 9. The problem geometry and the element mesh for the wire-drawing process.

properties of the layer are the mean values of the wire and the die at $20^{\circ} \mathrm{C}$. The finite element discretization consisted of 64 four-noded linear isoparametric elements. There are 180 displacement and 90 temperature degrees of freedom. The analysis has been performed in 425 increments, each $5 \cdot 10^{-6}$ seconds long. The die is spatial fixed, hence nodal points at the interface are consequently fixed in space; besides, no free surface motion occurs so the purely Eulerian method has been applied. The resulting temperature distribution is shown in Fig. 10.

The analysis has been continued until a steady state was almost achieved in the wire. In the die the steady-state temperature distribution is not yet achieved; therefore, the temperature distribution in the wire can change slightly. However continuing the analysis will probably produce no relevant, additional information. The process was fast, hence dissipation is more significant than conduction. By comparing the distribution of the equivalent plastic strain (Fig. 11) with the temperature distribution it can be seen that the influence of conduction is of less importance. The temperature changes are merely due to dissipation. 


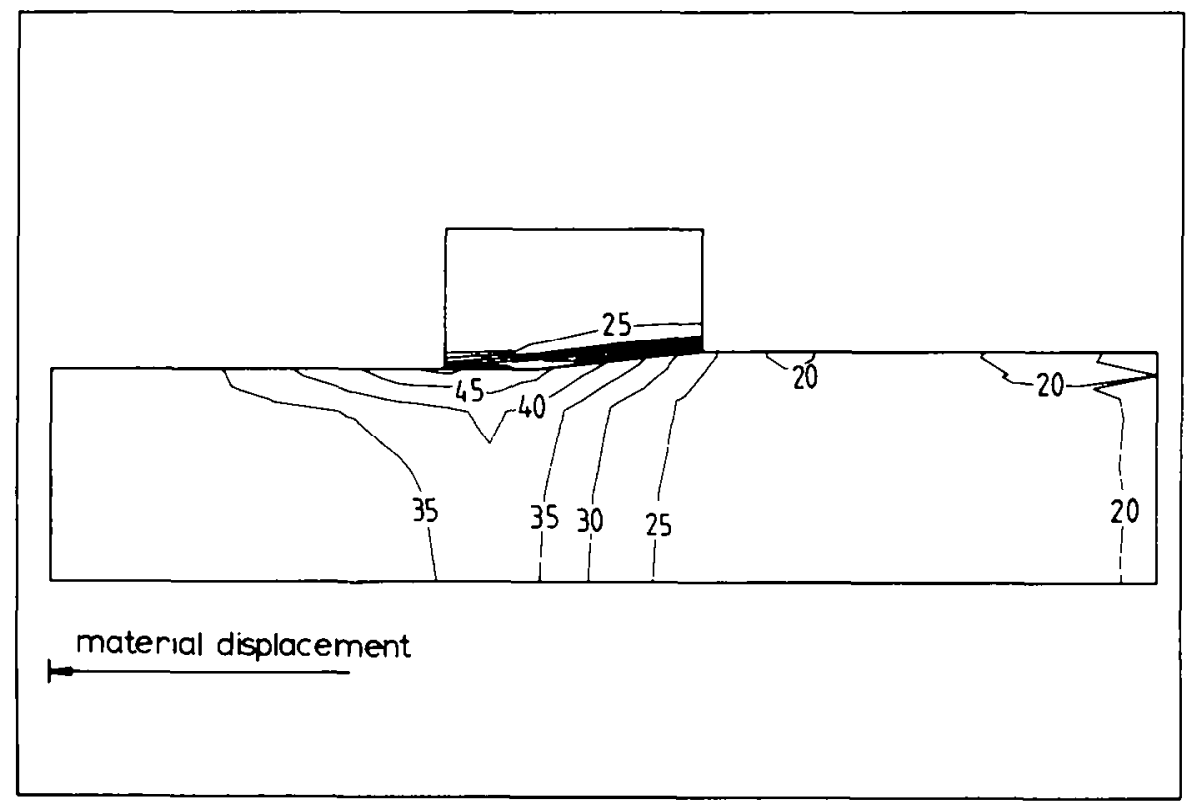

Fig. 10. The temperature distribution for the wire-drawing process.

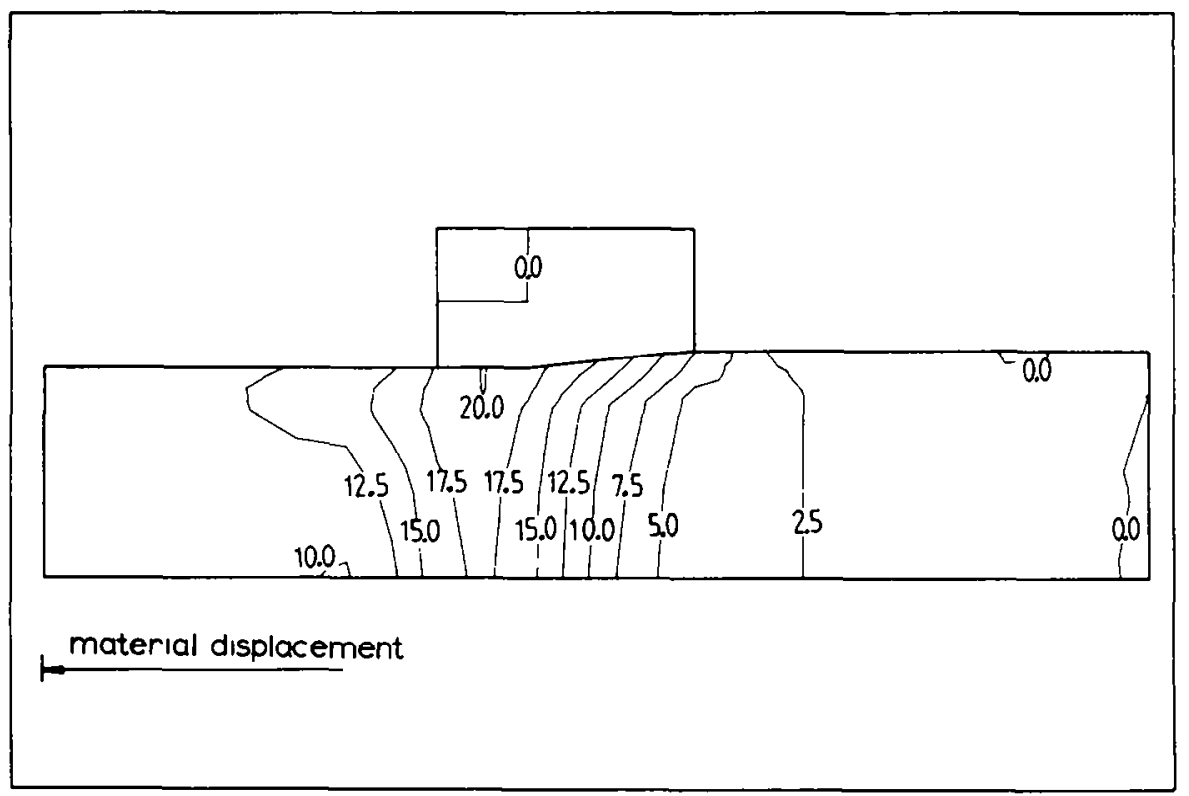

Fig. 11. The distribution of the equivalent plastic strain for the wire-drawing process (in percents). 


\section{Conclusion}

A combined Eulerian-Lagrangian finite element formulation for thermal mechanically coupled problems has been developed. The modification of the numerical integration avoids inaccurate temperature prediction in the case of fast deformation. The numerical analysis of the upsetting process shows satisfactory agreement with experimental results. With the knowledge of the material behaviour and the boundary conditions encountered in metalforming processes similar problems can be solved. An analysis of the wire-drawing process has been carried out, which shows reasonable results.

\section{References}

[1] J.H. Argyris and J.St. Doltsinis, On the natural formulation and analysis of large deformation coupled thermomechanical problems, Comput. Meths Appl. Mech. Engrg. 25 (1981) 195-253.

[2] J.H. Argyris, J.St. Doltsinis, P.M. Pimenta and H. Wüstenberg, Thermomechanical response of solids at high strains-natural approach, Comput. Meths Appl. Mech. Engrg. 32 (1982) 3-57.

[3] T.B. Wertheimer, Thermal mechanically coupled analysis in metal forming processes, in: Proceedings Internat. Conference on Numerical Methods in Industrial Forming (Pineridge, Swansea, U.K., 1982) 425-434.

[4] M.A. Biot, Variational Lagrangian-thermodynamics of nonisothermal finite strain mechanics of porous solids and thermomolecular diffusion, Internat. J. Solids and Structures 13 (1977) 579-597.

[5] J.F. Besseling, A thermodynamic approach to rheology, in: Proceedings IUTAM Symposium on Irreversible Aspects of Continuum Mechanics (Springer, Wien, 1968) 16-53.

[6] A.M.A. van der Heijden and J.F. Besseling, A large strain plasticity theory and the symmetry properties of its constitutive equations, University of Technology, Delft, LTM Rep. 726, 1982.

[7] Walter J. Moore, Physical Chemistry (Longman, London, 1976).

[8] J.C. Francken, Thermodynamica (Brill, Leyden, 1968), in Dutch.

[9] H. Parkus, Thermo-elasticity, Second revised and enlarged edition (Springer, Wien, 1976).

[10] W. Prager, Introduction to Mechanics of Continua (Ginn, New York, 1961).

[11] D.C. Drucker, Some implications of work hardening and ideal plasticity, Quart. Appl. Math. 7 (1950) 411-418.

[12] J. Huetink, Analysis of metal forming processes based on a combined Eulerian-Lagrangian finite element formulation, in: Proceedings Internat. Conference on Numerical Methods in Industrial Forming (Pineridge, Swansea, U.K., 1982) 501-509.

[13] O.C. Zienkiewicz, The Finite Element Method (McGraw-Hill, London, 1979).

[14] J.H. Wilkinson and C. Reinsch, Solution of real and complex systems of linear equations, in: F.L. Bauer, ed., Handbook for Automatic Computation, Vol. II. Linear Algebra (Springer, Berlin, 1971) 93-110.

[15] K.J. Bathe and E.L. Wilson, Numerical Methods in Finite Element Analysis (Prentice-Hall, Englewond Cliffs, NJ, 1976).

[16] H.D. Hibbitt, P.V. Marcal and J.C. Rice, A finite element formulation for large strain and large displacement, Internat. J. Solids and Structures 6 (1970) 1069-1086.

[17] R.M. McMeeking and J.C. Rice, Finite element formulation for large elastic plastic deformations, Internat. J. Solids and Structures 11 (1975) 601-616.

[18] Stiomak/THT, Materiaalkeuze in de Werktuigbouwkunde (Educaboek, Culemborg, 1978) in Dutch. 